\title{
Distal Radius Fractures: AAOS Appropriate Use Criteria Versus Actual Management at a Level I Trauma Center
}

\author{
James C. Kyriakedes', Eugene Y. Tsai', Douglas S. Weinberg', Charles C. Yu', \\ Harry A. Hoyen', Kevin Malone', and Blaine T. Bafus'
}

\begin{abstract}
Background: The aim of this study is to determine whether the American Academy of Orthopaedic Surgeons' (AAOS) Appropriate Use Criteria (AUC) for distal radius fractures correlates with actual treatment by orthopedic hand surgeons at a level I trauma center. Methods: ICD-9 codes were used to retrospectively identify patients who presented with wrist fractures over I year. Patients with isolated distal radius fractures were evaluated using the AAOS AUC application for distal radius fractures. Actual treatment was then compared with treatment recommended by the AUC. Results: Of the I I 2 patients, 64 (57\%) received treatment that matched the AAOS AUC recommendation as an "appropriate treatment." Actual management matched the AUC recommendation $100 \%, 7 \%$, and $50 \%$ of the time, for Arbeitsgemeinschaft für Osteosynthesefragen/Orthopaedic Trauma Association (AO/OTA) type A, B, and C fractures, respectively. Surgery was performed for type $A, B$, and $C$ fractures $30 \%, 7 \%$, and $50 \%$ of the time, respectively. For type B fractures, only the 2 cases that were managed operatively were in agreement with the AUC. For type C fractures, increased patient age (57 years and older) was significantly associated with nonoperative treatment decisions. Surgeon decisions for nonoperative treatment were in agreement with the AUC recommendations $40 \%$ of the time, whereas surgeon decisions for surgery matched the AUC recommendations $97 \%$ of the time. Conclusions: We found low agreement between actual treatment decisions and the AUC-recommended "appropriate" treatments, especially for the type B and C fractures that were managed nonoperatively. The AUC favors surgery for all intra-articular fractures, while we emphasized age and fracture displacement in our decision-making process.
\end{abstract}

Keywords: AAOS, Appropriate Use Criteria, distal radius fracture, fragility fracture, clinical practice guidelines

\section{Introduction}

Distal radius fractures are one of the most commonly sustained fractures in the adult population with an incidence of about 643,000 per year in the United States. ${ }^{8,9,13}$ In the Medicare population alone, there is an estimated $\$ 1.1$ billion annual cost to treat osteoporotic fractures, $18 \%$ of which are distal radius fractures. ${ }^{7,26,27}$ The proper treatment of various types of distal radius fractures has long been an issue of debate, and one which continues to evolve with new research and surgical techniques. In the Medicare population, treatment increased from 3\% open reduction internal fixation (ORIF) in 1996 to $16 \%$ ORIF in $2005 .^{7}$ It has recently been shown that surgeons younger than 40 years of age are more likely than older surgeons to perform ORIF. ${ }^{31}$ Given the high incidence of this fracture, which is expected to increase as our elderly population continues to expand, ${ }^{27,30}$ and increasing use of operative management, guidelines on the proper treatment of distal radius fractures have far-reaching effects.
In 2013, the American Academy of Orthopaedic Surgeons (AAOS) released the first of its Appropriate Use Criteria (AUC) based on its 2009 Clinical Practice Guideline for the treatment of distal radius fractures. ${ }^{21}$ The AUC added clinical expertise from physicians across multiple medical specialties. The Writing Panel created 240 clinical scenarios based on different combinations of types of patients and injuries that clinicians are likely to encounter. The Voting Panel individually rated the 10 treatment options as "appropriate," "may be appropriate," or "rarely appropriate," by "using their best clinical judgment, taking into consideration

'Department of Orthopaedic Surgery, MetroHealth Medical Center, Case Western Reserve University, Cleveland, $\mathrm{OH}$, USA

\section{Corresponding Author:}

James C. Kyriakedes, Department of Orthopaedic Surgery, MetroHealth Medical Center, 2500 MetroHealth Drive, Cleveland, OH 44109, USA. Email: kyriakjc@gmail.com 
the available evidence, for an average patient presenting to an average physician at an average facility." The AAOS then developed a web-based application for surgeons to use in applying the AUC in clinical practice. ${ }^{2}$

The purpose of this study was to compare the treatments performed by 5 hand fellowship-trained orthopedic surgeons at a high-volume urban level I trauma center with the recommendations endorsed by the AUC, as a test of the application. We hypothesized that our surgeons would have low agreement rates with the AUC, primarily with regard to management of nondisplaced or minimally displaced fractures and the AUC application's lack of inclusion of fracture displacement as a variable.

\section{Materials and Methods}

With approval from our institutional review board, we retrospectively reviewed the charts of all patients who presented to our institution, which is a high-volume urban level I trauma center, with a wrist fracture ICD-9 code (813.41814.01) between August 1, 2011, and August 1, 2012. Only patients treated by one of the hand fellowship-trained orthopedic surgeons with isolated distal radius fractures and those with pretreatment and posttreatment radiographs were included.

Exclusion criteria included pediatric patients who were not skeletally mature $(\mathrm{n}=194)$, those erroneously coded as a distal radius fracture $(\mathrm{n}=154)$, those who were not managed by an orthopedic hand surgeon $(n=130)$, those whose initial presentation was outside of our study period $(n=92)$, those who had sustained other significant injuries concomitantly with their distal radius fracture $(\mathrm{n}=66)$, those who did not follow up in a orthopedic hand surgery clinic $(\mathrm{n}=$ 37), those with incomplete pretreatment and posttreatment radiographs $(n=35)$, those whose initial presentation was 4 or more weeks after injury $(n=6)$, and patients who refused operative treatment if recommended by the attending surgeon $(\mathrm{n}=3)$.

Of the 829 patients who presented to our medical center with an ICD-9 code for a wrist fracture, 112 met our inclusion criteria. The radiographs for each of these patients were then evaluated by an orthopedic surgeon and assigned an AO/OTA (Arbeitsgemeinschaft für Osteosynthesefragen/ Orthopaedic Trauma Association) classification. ${ }^{20}$ Information on each patient's mechanism of injury (high- vs lowenergy), activity level (normal, high functional, independent, or homebound), American Society of Anesthesiologists (ASA) status (1-3 or 4), and the presence of select associated injuries (median nerve injury, open fracture, or ipsilateral injury) were then extracted via chart review. These are the variables used in the AUC application. Also extracted were the patient's age and the treating surgeon's assessment of fracture displacement, or other significant measures of distal radius radiographic alignment, which led to the treatment decision.

As written in the AUC application, high-energy fractures involve "high compression forces" and "assume significant displacement and comminution." The AUC provided examples such as falls from greater than standing height, motor vehicle collisions, and industrial accidents with high velocity impacts. Low-energy mechanisms involve "chronic conditions that weaken the strength of the bone and low velocity at impact results in bending forces" and "assume minimal comminution and displacement." Activity levels were divided into: (1) normal ("activities of daily living [ADLs] without assistance"); (2) high functional ("substantial stress/strain on wrist on regular basis, such as a high-level athlete or heavy laborer"); (3) independent ("routinely completes ADLs with assistance of ambulation devices"); and (4) homebound ("require human assistance to leave home"). ASAs were separated into 2 categories: (1) ASA 1 to 3 ("normal healthy patient" to one "with severe systemic disease"); and (2) ASA 4 ("patient with severe systemic disease that is a constant threat to life"). The data for each of the patients included in the study were then entered into the distal radius AUC application. ${ }^{2}$

A post hoc power analysis was performed to determine the minimum number of patients required. Assuming a significance level (alpha) of 0.05 , power (beta) of 0.80 , and effect size (delta) of 0.20 , with 4 significant predictors assumed, 69 patients were deemed necessary for analysis. Normality was assessed with the Shapiro-Wilk test, and Q-Q plots were generated. Continuous variables were assessed with the independent samples $t$ test or MannWhitney $U$ test, where appropriate. Categorical variables were compared with the Fisher exact or chi-square test. Post hoc comparisons, when necessary, were made with $Z$ testing (Bonferroni method, $\alpha \leq 0.05$ ). ${ }^{1}$ Binary logistic regression was used to determine independent predictors of operating criterion. The dependent variables were agreement with AUC guidelines (yes/no). Independent variables included age, gender, mechanism of injury, and ASA status. Stepwise binomial logistic regression was performed on covariates with $P<.10$ in univariate analysis. Significance was set at a $P$ value less than or equal to .05 .

\section{Results}

Our study sample included 112 patients (35 males and 77 females) with an average age of 50 years. Ninety-four of 112 fractures (84\%) were low-energy, and only 3 patients had an ASA of 4 . There was a relatively even distribution of AO/OTA fracture types with 42 of $112(37 \%)$ type A, 30 of $112(27 \%)$ type $\mathrm{B}$, and 40 of $112(36 \%)$ type $\mathrm{C}$ fractures. Seventy-eight of 112 patients ( $70 \%$ ) were treated nonoperatively, whereas 34 of $112(30 \%)$ were treated operatively 
Table I. Distribution of Patients Among 10 Possible AUC Treatment Types.

\begin{tabular}{lc}
\hline Treatment type & Number of patients \\
\hline Immobilization without reduction & 52 \\
Immobilization with reduction & 26 \\
Percutaneous pinning & 4 \\
Volar locking plate & 24 \\
Dorsal plate & 2 \\
Fragment-specific fixation & 3 \\
Intramedullary nail & 0 \\
Spanning external fixator & 1 \\
Nonspanning external fixator & 0 \\
Distraction plate & 0
\end{tabular}

Note. AUC $=$ Appropriate Use Criteria.

Table 2. Average Patient Age (in Years) and Standard Deviation of Operative and Nonoperative Management for Various AO/OTA Distal Radius Fracture Types.

\begin{tabular}{lcccc}
\hline $\begin{array}{l}\text { AO/OTA } \\
\text { fracture type }\end{array}$ & $\begin{array}{c}\text { Total } \\
(\mathrm{n}=112)\end{array}$ & $\begin{array}{c}\text { Nonoperative } \\
\text { (age in years) }\end{array}$ & $\begin{array}{c}\text { Operative } \\
(\text { age in years) }\end{array}$ & $\begin{array}{c}\text { Significance } \\
\left(P \text { values }{ }^{\mathrm{a}}\right)\end{array}$ \\
\hline A & $12 / 42(38 \%)$ & $54.0 \pm 20.4$ & $49.6 \pm 10.3$ & .485 \\
B & $2 / 30(27 \%)$ & $46.9 \pm 14.4$ & $46.0 \pm 8.5$ & .935 \\
C & $20 / 40(36 \%)$ & $58.6 \pm 13.5$ & $39.3 \pm 15.6$ & $<.001$ \\
\hline
\end{tabular}

Note. $\mathrm{AO} / \mathrm{OTA}=$ Arbeitsgemeinschaft für Osteosynthesefragen/Orthopaedic Trauma Association.

${ }^{a}$ The $P$ values in this table are comparisons between the average patient ages of operative and nonoperative groups.

(Table 1). Rates of surgery were 12 of 42 (29\%) for type A fractures, 2 of $30(7 \%)$ for type B fractures, and 20 of 40 $(50 \%)$ for type $\mathrm{C}$ fractures.

Of the 112 patients, $64(57 \%)$ received treatment that would have matched the AAOS AUC recommendation classification as an "appropriate" treatment. By grouping fractures into AO/OTA type, we found that actual management matched the AUC recommendation in $100 \%$ of the cases classified as type A (42 of 42), $7 \%$ of the cases classified as type B ( 2 of 30 ), and $50 \%$ of the cases classified as type C ( 20 of 40 ). For type B fractures, the 2 cases in agreement with the AUC were the 2 that were managed operatively.

The results of binomial logistic regression suggest that for type $\mathrm{C}$ fractures, patients 57 years and older were less likely than those younger than 57 to undergo operative treatment (odds ratio, $0.114 ; 95 \%$ confidence interval, $0.019-0.670 ; P=.016$ ). In addition, subgroup analysis of fractures by age found type $\mathrm{C}$ fractures to be operatively managed in a significantly younger cohort of patients $(39 \pm$ 16 years), as compared with those managed nonoperatively (59 \pm 14 years, $P<.001$; Table 2$)$. Similarly, AUC agreement rates varied significantly by age for type $\mathrm{C}$ fractures $(42 \pm 16$ years for AUC agreement vs $56 \pm 16$ years for AUC disagreement, $P=.009$; Table 3 ).
Table 3. Average Patient Age (in Years) and Standard Deviation for Agreement and Disagreement With AUC for Various AO/OTA Distal Radius Fracture Types.

\begin{tabular}{lcccc}
\hline $\begin{array}{l}\text { AO/OTA } \\
\text { fracture type }\end{array}$ & $\begin{array}{c}\text { Total } \\
(\mathrm{n}=112)\end{array}$ & $\begin{array}{c}\text { Agreement } \\
\text { with AUC } \\
\text { (age in years) }\end{array}$ & $\begin{array}{c}\text { Disagreement } \\
\text { with AUC } \\
(\text { age in years })\end{array}$ & $\begin{array}{c}\text { Significance } \\
\left(P \text { values }^{\mathrm{a}}\right)\end{array}$ \\
\hline $\mathrm{A}$ & $42 / 42(38 \%)$ & - & $52.7 \pm 18.1$ & - \\
$\mathrm{B}$ & $2 / 30(27 \%)$ & $46.9 \pm 14.4$ & $46.0 \pm 8.5$ & .935 \\
C & $20 / 40(36 \%)$ & $56.0 \pm 16.4$ & $41.9 \pm 16.2$ & .009 \\
\hline
\end{tabular}

Note. AUC = Appropriate Use Criteria. AO/OTA = Arbeitsgemeinschaft für Osteosynthesefragen/ Orthopaedic Trauma Association.

${ }^{a}$ The $P$ values in this table are comparisons between the average patient ages with agreement or disagreement with the AUC treatment recommendation.

The choice of nonoperative fracture treatment was in agreement with the AUC recommendation only $40 \%$ of the time (31 of 78, $P=.002$ ). A decision to recommend surgical treatment matched the AUC recommendation $97 \%$ of the time (33 of 34, $P<.001$ ). Actual management decisions that matched AUC recommendations were 50 times more likely to have been for operative fixation, as compared with when decisions did not match the AUC recommendations $(P<.0001)$. When there was agreement between the actual management and the AUC recommendation for management, the injury was 4.5 times more likely to be high-energy than low-energy $(P=.01)$.

\section{Discussion}

The development of a clinical tool such as the AUC, which represents best clinical practice as recommended by evidence-based medicine, is an invaluable resource for clinicians. In addition to affecting clinical practice, the applicability of clinical tools such as the AUC has potentially far-reaching economic and medicolegal effects. The direct cost of nonoperative versus operative management differs vastly. ${ }^{27}$ In our increasingly cost-conscious health care environment, the decision to manage distal radius fractures, a very common injury, operatively instead of nonoperatively cannot be taken lightly. While we agree with the concept of the AUC as being a helpful clinical tool, our finding of only $57 \%$ agreement between our observed practice and the AUC recommendations is surprising.

The discrepancy occurred with type $\mathrm{B}$ and type $\mathrm{C}$ fractures, where there was only $7 \%$ and $50 \%$ agreement, respectively. The AUC application was much more aggressive in advocating operative management of intra-articular fractures, whereas in practice, we only managed $7 \%$ and $50 \%$ of type $\mathrm{B}$ and type $\mathrm{C}$ fractures with surgery, respectively. Interrogating the AUC application with various actual and hypothetical clinical scenarios, we found that the application advocates for operative management for all type B and type $\mathrm{C}$ fractures, except in patients with ASA 4 ("severe systemic 
disease that is a constant threat to life") and homebound ("needing human assistance to leave home"), regardless of mechanism of injury (i.e. low- vs high-energy). While determining ASA status and activity level is helpful in deciding to withhold surgery in those extreme scenarios, the healthy and active, while osteopenic or osteoporotic, elderly patients with distal radius fractures were encountered much more frequently in our practice. Actual treatment recommendations at our center were dependent on age for type $\mathrm{C}$ fractures and intra-articular fracture displacement, as determined at the surgeon's discretion.

Our older patients (greater than 57 years of age) with type $\mathrm{C}$ fractures tended to be managed nonoperatively, and our patients 56 years and younger tended to be managed operatively (Tables 2 and 3). Patient age is not assessed by the AUC. It has previously been shown that increasing patient age is an independent risk factor for secondary displacement and instability after distal radius fracture closed reduction. ${ }^{17,23}$ However, a number of recent studies have determined that operative treatment of distal radius fractures in elderly patients, including those who are highly active, is associated with greater complications without significant differences in functional outcomes or pain as compared with nonoperative treatment. ${ }^{3-5,11,18,19,22,29,32}$ In a 2016 prospective cohort of 129 high-functioning patients older than 55 years, 1-year functional outcome scores were affected not by whether their fracture was managed nonoperatively or operatively but by the degree of persistent intraarticular displacement or radial shortening after either treatment. ${ }^{18}$ Therefore, nonoperative management is a valid treatment option in these patients, regardless of the $\mathrm{AO} /$ OTA fracture type.

Fracture displacement also played a role in our surgeons' decision making, with nondisplaced or minimally displaced fractures likely to be managed nonoperatively. The AUC attempts to capture the amount of displacement of the fracture by taking into account mechanism of injury. Even when an intra-articular fracture was low-energy, which the AUC describes as "assuming minimal displacement," the application recommended only operative management as "appropriate" unless the patient was ASA 4 or homebound (Figure 1). While mechanism of injury has an impact on the nature of the fracture and can affect the inherent stability of the fracture, it is not a perfect proxy for fracture displacement. The amount the fracture is assumed to alter normal wrist mechanics and the amount of articular step-off tends to play large roles in clinical decision making, as the purpose of fracture treatment is to maximize functional outcome and to minimize the potential for future joint arthrosis. Knirk and Jupiter, ${ }^{16}$ in their frequently cited 1986 study, popularized the idea that intra-articular incongruity of greater than $2 \mathrm{~mm}$ is an indication for surgery based on the risk for developing future joint arthrosis. ${ }^{15}$

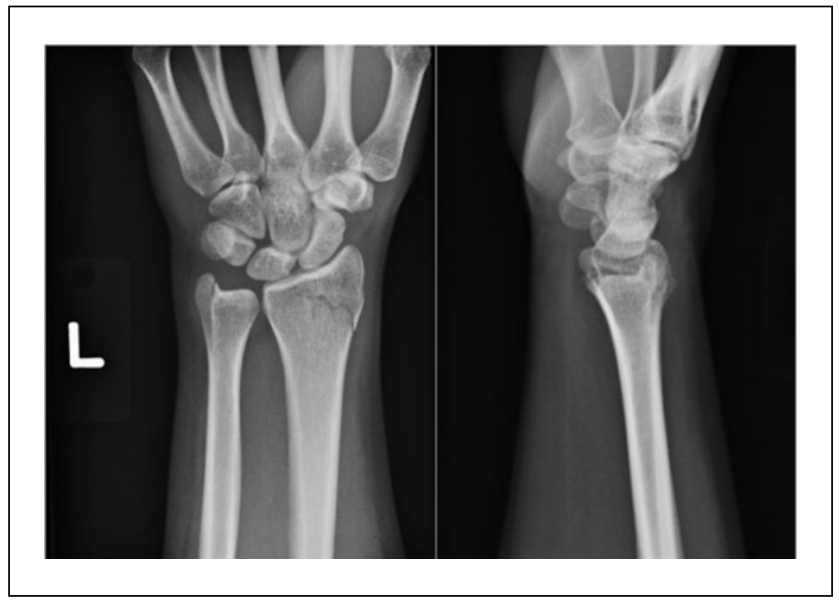

Figure I. Example of a healthy 28-year-old who sustained a low-energy AO/OTA type B fracture for which the AUC recommended operative treatment. When entered into the AUC, immobilization without reduction, which is what was performed, was deemed "rarely appropriate," its lowest recommendation. The patient went on to successful painless union of her fracture.

Note. AO/OTA = Arbeitsgemeinschaft für Osteosynthesefragen/ Orthopaedic Trauma Association; AUC = Appropriate Use Criteria.

The effect of radiographic joint arthrosis on functional outcomes continues to be a source of controversy, however, as more recently published studies have not shown a significant effect on outcome. ${ }^{12,28}$ High-quality, long-term, prospective randomized controlled studies have yet to be performed to settle this controversy; therefore, it is unclear whether a clinical application that does not take fracture displacement into account recommends best practices.

The AUC's use of mechanism of injury instead of fracture displacement was likely secondary to the inherent difficulties of accurately and consistently measuring fracture displacement on radiographs. ${ }^{6,24}$ Calibrated radiographs and/or computerized tomography (CT) scans, which could be used to more accurately gauge displacement, are not regularly obtained for distal radius fractures. CT scans may incur unacceptably high increases in cost and radiation exposure, although previous studies have demonstrated their utility. ${ }^{14,25}$

There are limitations to this study. This was a retrospective study that did not attempt to assess long-term followup. Future directions involve assessing radiographic and functional outcomes after prolonged follow-up. Doing so might elucidate whether agreement or disagreement with the AUC recommendation resulted in any long-term differences in outcomes. Our study was limited to one institution, which is a high-volume level I urban trauma center. However, the fact that our greatest discrepancies were with low-energy, intra-articular fractures in elderly patients, 
which one would assume constitute an even larger proportion of the fractures treated at nontrauma centers, calls into greater question the universal applicability of the tool.

The AAOS AUC for treatment of full-thickness rotator cuff tears was recently evaluated, and $79 \%$ agreement was observed between actual management of 134 patients and the AUC recommendations of "appropriate" treatments. ${ }^{10}$ Despite a concordance with the AUC described as "excellent," the authors also found significant improvement in the functional outcome scores of all patients at prolonged follow-up, even when management was not in agreement with the AUC recommendations. The lack of inclusion of patient age was also listed as a limitation to the AUC for rotator cuff tears.

We conclude that the AAOS AUC favors surgery for all intra-articular distal radius fractures. Actual treatment recommendations at our center were dependent on age for type $\mathrm{C}$ fractures and intra-articular fracture displacement. Treating clinicians may find a modified AUC that takes into account age and intra-articular fracture displacement more useful.

\section{Ethical Approval}

Institutional review board approval was granted prior to beginning this retrospective chart review.

\section{Statement of Human and Animal Rights}

This article does not contain any studies with human or animal subjects.

\section{Statement of Informed Consent}

Informed consent was not required.

\section{Declaration of Conflicting Interests}

The author(s) declared the following potential conflicts of interest with respect to the research, authorship, and/or publication of this article: H.A.H. lists disclosures of stock or stock options in AxoGen Inc, a paid consultant and paid presenter for Stryker, and a paid consultant for Lima. There are no other potential conflicts of interest, and the authors do not feel that the potential perceived conflicts described here affected the methods, results, or conclusions of this study.

\section{Funding}

The author(s) received no financial support for the research, authorship, and/or publication of this article.

\section{References}

1. Abdi H. Bonferroni and Šidák corrections for multiple comparisons. In: Salkind NJ, ed. Encyclopedia of Measurement and Statistics. Thousand Oaks, CA: Sage; 2007.

2. American Academy of Orthopaedic Surgeons (AAOS). https://aaos.webauthor.com/go/auc/auc.cfm?auc_id=224789. Accessed March 2, 2015.
3. Anzarut A, Johnson JA, Rowe BH, et al. Radiologic and patient-reported functional outcomes in an elderly cohort with conservatively treated distal radius fractures. J Hand Surg Am. 2004;29(6):1121-1127.

4. Arora R, Lutz M, Deml C, et al. A prospective randomized trial comparing nonoperative treatment with volar locking plate fixation for displaced and unstable distal radial fractures in patients sixty-five years of age and older. J Bone Joint Surg Am. 2011;93(23):2146-2153.

5. Bartl C, Stengel D, Bruckner T, et al. The treatment of displaced intra-articular distal radius fractures in elderly patients. Dtsch Arztebl Int. 2014;111(46):779-787.

6. Catalano LW, Barron OA, Glickel SZ. Assessment of articular displacement of distal radius fractures. Clin Orthop Relat Res. 2004;423:79-84.

7. Chung KC, Shauver MJ, Birkmeyer JD. Trends in the United States in the treatment of distal radial fractures in the elderly. J Bone Joint Surg Am. 2009;91(8):1868-1873.

8. Chung KC, Spilson SV. The frequency and epidemiology of hand and forearm fractures in the United States. J Hand Surg Am. 2001;26(5):908-915.

9. Court-Brown CM, Aitken SA, Forward et al. The epidemiology of fractures. In: Bucholz RW, Court-Brown CW, Heckman JD, Tornetta P, eds. Rockwood and Green's Fractures in Adults. 7th ed. Philadelphia, PA: Lippincott Williams \& Wilkins; 2010:53-84.

10. Cowan JB, Bedi A, Carpenter JE, et al. Evaluation of American academy of orthopaedic surgeons appropriate use criteria for the management of full-thickness rotator cuff tears. J Shoulder Elbow Surg. 2016;25(7):1100-1106.

11. Diaz-Garcia RJ, Oda T, Shauver MJ, et al. A systematic review of outcomes and complications of treating unstable distal radius fractures in the elderly. J Hand Surg Am. 2011;36(5):824-835.

12. Goldfarb C, Rudzki JR, Catalano LW, et al. Fifteen-year outcome of displaced intra-articular fractures of the distal radius. J Hand Surg Am. 2006;31(4):633-639.

13. Hammert WC, Kramer RC, Graham B, et al. AAOS appropriate use criteria: treatment of distal radius fractures. $\mathrm{J} \mathrm{Am} \mathrm{Acad}$ Orthop Surg. 2013;21(8):506-509.

14. Harness NG, Ring D, Zurakowski D, et al. The influence of three-dimensional computed tomography reconstructions on the characterization and treatment of distal radial fractures. $J$ Bone Joint Surg Am. 2006;88(6):1315-1323.

15. Haus BM, Jupiter JB. Intra-articular fractures of the distal end of the radius in young adults: reexamined as evidencebased and outcomes medicine. J Bone Joint Surg Am. 2009;91(12):2984-2991.

16. Knirk JL, Jupiter JB. Intra-articular fractures of the distal end of the radius in young adults. J Bone Joint Surg Am. 1986;68(5):647-659.

17. Lafontaine M, Hardy D, Delince P. Stability assessment of distal radius fractures. Injury. 1989;20:208-210.

18. Larouche J, Pike J, Slobogean GP, et al. Determinants of functional outcome in distal radius fractures in highfunctioning patients older than 55 years. J Orthop Trauma. 2016;30(8):445-449.

19. Lutz K, Yeoh KM, MacDermid JC, et al. Complications associated with operative versus nonsurgical treatment of distal 
radius fractures in patients aged 65 years and older. $J$ Hand Surg Am. 2014;39(7):1280-1286.

20. Marsh JL, Slongo TF, Agel J, et al. Fracture and dislocation classification compendium-2007: Orthopaedic Trauma Association classification, database and outcomes committee. J Orthop Trauma. 2007:21(10)(suppl):S1-S133.

21. Murray J, Gross L. Treatment of distal radius fractures. $J$ Am Acad Orthop Surg. 2013;21(8):502-505.

22. Nelson GN, Stepan JG, Osei DA, et al. The impact of patient activity level on wrist disability after distal radius malunion in older adults. J Orthop Trauma. 2015;29(4):195-200.

23. Nesbitt KS, Failla JM, Les C. Assessment of instability factors in adult distal radius fractures. J Hand Surg Am. 2004:29(6):1128-1138.

24. O'Malley MP, Rodner C, Ritting A, et al. Radiographic interpretation of distal radius fractures: visual estimations versus digital measuring techniques. Hand (N Y). 2014;9(4):488-493.

25. Pruitt DL, Gilula LA, Manske PR, et al. Computed tomography scanning with image reconstruction in evaluation of distal radius fractures. J Hand Surg Am. 1994;19(5):720-727.

26. Ray NF, Chan JK, Thamer M, et al. Medical expenditures for the treatment of osteoporotic fractures in the United States in 1995: report from the National Osteoporosis Foundation. J Bone Miner Res. 1997;12:24-35.

27. Shauver MJ, Yin H, Banerjee M, et al. Current and future national costs to Medicare for the treatment of distal radius fracture in the elderly. J Hand Surg Am. 2011;36(8):1282-1287.

28. Souer JS, Lozano-Calderon SA, Ring D. Predictors of wrist function and health status after operative treatment of fractures of the distal radius. J Hand Surg Am. 2008;33(2):157-163.

29. Synn AJ, Makhni EC, Makhni MC, et al. Distal radius fractures in older patients: is anatomic reduction necessary? Clin Orthop Relat Res. 2009;467(6):1612-1620.

30. United States Census Bureau. Projections of the population by sex and age for the United States: 2015 to 2060. http:// www.census.gov/population/projections/data/national/2014/ summarytables.html. Accessed July 24, 2016.

31. Waljee JF, Zhong L, Shauver MJ, et al. The influence of surgeon age on distal radius fracture treatment in the United States: a population-based study. J Hand Surg Am. 2014:39(5):844-851.

32. Young BT, Rayan GM. Outcome following nonoperative treatment of displaced distal radius fractures in low-demand patients older than 60 years. J Hand Surg Am. 2000;25(1):19-28. 